\title{
Merkmale des Fachsprachengebrauchs im Wasserballunterricht
}

\begin{abstract}
Tímea H. Tomesz
Aspects of the use of terminology in water polo instruction Abstract

Just as in other domains of knowledge acquisition, verbal skills have a key role in sports instruction as well. In the course of preparation as well as during competitions and matches, it is crucial for the efficiency of tactical-strategic discourse that young athletes possess terminological knowledge about their disciplines that is adequate for their age and cognitive development. When the child is in possession of appropriate terminological knowledge, his/her sport-specific motor skills can be developed in a more targeted way. The goal of my survey was to map the terminological knowledge of children learning to play water polo and thereby to explore aspects of terminology acquisition. The paper first discusses changes in terminological vocabulary and touches on general features of sports instruction. Subsequently, it reports on the results of a questionnaire survey, highlighting the main features of how terminology is used in water polo instruction.
\end{abstract}

Keywords: terminological communication; child language; sports instruction; efficiency; water polo

Schlüsselwörter: Terminologische Kommunikation; Sportpädagogik; Kindersprache; Effizienz; Wasserball

Subject-Affiliation in New CEEOL: Language and Literatura - Applied Linguistics Sociolinguistics

DOI: 10.36007/eruedu.2021.4.44-54

\section{Einführung}

Obwohl im Sportunterricht der Schwerpunkt auf den motorischen Aktivitäten liegt und die Wirksamkeit des Unterrichts an die durch objektive Parameter messbare Leistung gebunden ist, erfolgt das Lernen und die Umsetzung von Aktivitäten in einem Kommunikationsprozess mit Hilfe von Kommunikationsmitteln und -methoden. Daher spielt die Verbalität, wie andere Wissenserwerbs- und Lernprozesse, eine wichtige Rolle im Sportunterricht.

In der Vorbereitungsphase, bei Wettkämpfen und Spielen, ist es für die Effizienz des taktisch-strategischen Diskurses von grundlegender Bedeutung, dass ein Sportler Begriffe des Fachvokabulars seiner Sportart beherrscht, die seinem Alter und seiner kognitiven Entwicklung entsprechen. Wenn das Kind über angemessene Kenntnisse der Fachsprache verfügt, kann die Entwicklung seiner Bewegung gezielter gefördert werden. 
Das Ziel meiner Forschung war es, die fachsprachlichen Kenntnisse von Kindern, die Wasserball spielen, zu erfassen sowie die Merkmale und den Prozess des Fachsprachenerwerbs zu untersuchen. Mit diesen Zielen vor Augen klärt die Studie zunächst die für die Erforschung des Fachsprachengebrauchs erforderlichen Grundbegriffe, diskutiert die Merkmale, die die Herausbildung des Fachvokabulars und die Veränderungen im Wortschatz kennzeichnen (2), beschreibt die Besonderheiten der Sportpädagogik (3) sowie den Forschungsgegenstand und die -methoden (4) und stellt dann die Ergebnisse vor (5).

\section{Veränderungen im Wortschatz, Herausbildung des Fachvokab- ulars}

Ein primärer Faktor bei der Entwicklung des Wortschatzes ist das soziale Umfeld, d.h. die verfügbaren sprachlichen Muster (Neuberger 2008). Das sprachliche Umfeld vor der institutionellen Bildung wird von der Familie (insbesondere der Mutter) geschaffen und geprägt. In den frühen Stadien der Sprachentwicklung kommt es zu einer Verschiebung zwischen der Sprachrezeption und der -produktion, weil das Kind mehr versteht, als es selbst sprachlich äußern kann. Typisch für diesen Zeitraum (Alter 10 bis 15 Monate, Phase der Einwortsätze) ist auch die doppelte Speicherung, d.h. im mentalen Lexikon wird das jeweilige Wort mit zwei verschiedenen Lautketten gespeichert: einer konventionellen (Erwachsenensprache) und einer kindeseigenen (Kindersprache), z.B. ung. „babakocsi“ („Kinderwagen“) „bacsicsi“, „,bukósisak“ (,Sturzhelm“) - „butusisi“.

Die erste sprunghafte Veränderung im Wortschatz beginnt mit der institutionalisierten Bildung (Kinderkrippe, Kindergarten). Obwohl es recht schwierig ist, die Größe des Wortschatzes objektiv zu messen, ist eins sicher: sein Umfang und seine Qualität werden sowohl durch das soziale Leben als auch durch die neuen Gemeinschaften beeinflusst. Studien zeigen, dass die Sprachentwicklung bis zum Alter von eineinhalb Jahren langsamer verläuft und sich dann abrupt verändert (Neuberger 2017). Zu Beginn des Schulalters (6 bis 7 Jahre) ist aufgrund der Entwicklung kognitiver Fähigkeiten und des neuen sozialen Umfelds eine sprunghafte Erweiterung des Wortschatzes zu beobachten, und es kommt zu einer Abspaltung und kontinuierlichen Erweiterung des Fachvokabulars. In diesem Alter sind sich Kinder bereits über die Bedeutung von Fachwörtern im Klaren und kennen auch deren mögliche Zusammenhänge (Gósi 2005).

Parallel zur kognitiven Aktivität und der sprachlichen Entwicklung etabliert sich allmählich ein Sprachbewusstsein, das die Trennung von Bedeutung und Form ermöglicht (Adamikné 2006, 20). Während der konzeptuellen Entwicklung geht das Kind allmählich von einer auf direkten Erfahrungsinformationen basierten Konzeptualisierung zu abstrakteren, sprachlich strukturierten Begriffen über (Csépe és mtsai 2007, 296). Ein spezifischer Fall der Bedeutungs- und Worterschließung liegt vor, wenn ein Kind die Bedeutung eines Lexems nicht mehr anhand des Kontextes lernt, sondern sie definiert bekommt (Bóna-Imre 2017, 186-187). 


\section{Entwicklung des Fachvokabulars}

Obwohl die verbale Reife, das Vorhandensein bestimmter Kommunikationsfähigkeiten als Voraussetzung für den Schuleintritt gilt, kommt der Erweiterung des Wortschatzes, dem Erwerb spezifischer Sprachformen, der grammatikalisch korrekten Sprache und der differenzierten Formulierung eine entscheidende Rolle in der Kommunikationserziehung von Grundschulkindern zu. Darüber hinaus legt der Nationale Grundlehrplan (NAT) bei der Definition der Inhalte der einzelnen Bildungsbereiche im öffentlichen Bildungswesen auch das Fachvokabular des jeweiligen Bildungsbereichs (2012) fest. Demnach bilden die Kenntnis der Fachsprache und die Erweiterung des Fachwortschatzes einen Teil der Entwicklung der muttersprachlichen Kommunikationskompetenz.

Die Fachsprache nährt sich aus der Gemeinsprache, sie wird als Sprachgebrauch durch Fachleute für bestimmte Zwecke verstanden, sie wiederspiegelt in klarer und eindeutiger Weise einen bestimmten Teil der Wirklichkeit und ist eine charakteristische Ausprägung der mündlichen und schriftlichen Kommunikation (Kurtán 2003). Die Mitglieder einer fachsprachlichen Gemeinschaft können sich unterschiedlich ausdrücken, je nachdem, ob sie mit Adressaten mit dem gleichen beruflichen Hintergrund, Vertretern anderer Disziplinen oder Laien kommunizieren, und je nach dem Alter der Personen, an die sich die Kommunikation richtet. Darüber hinaus wird der Sprachgebrauch natürlich durch den Ort, den Zweck und das Medium der Kommunikation sowie durch die bestehenden Konventionen bestimmt (Kurtán 2011, 9).

Obwohl es in der Fachliteratur nicht einheitlich geklärt ist, ob die Sportsprache als Fachsprache oder eher als Gruppen- oder gar als Hobbysprache gilt, ist ihre Klassifizierung als Fachsprache am weitesten verbreitet (Mátis 2009, 26). Verstärkt wird dies durch die Tatsache, dass ihr Gebrauch weitgehend auf eine Berufsgruppe in der Gesellschaft bezogen ist, d.h. als Sprachvariante zum Ausdruck von Sprechabsichten für bestimmte Zwecke verwendet wird (Fóris 2003, 28). Ein weiteres Merkmal der Sportsprache ist, dass sie, wie andere Fachsprachen auch, der sprachlichen Logik der Gemeinsprache folgt, indem sie ein mit dem Gegenstand und der Funktion des jeweiligen Fachgebietes zusammenhängendes Vokabular verwendet. Ihr Erwerb ist ein bewusster Lernprozess und sie wird von den Mitgliedern kleinerer und größerer Berufsgruppen genutzt, um den verbalen Kontakt im Fachgebiet zu erleichtern (vö. Ablonczyné Mihályka 2006, 23-24).

Kovács und Osvald halten es für wichtig, Sachwissen und Fachsprachenwissen voneinander abzugrenzen: ersteres dient als Marker für enzyklopädisches Sachwissen, letzteres wird als Kenntnis der relevanten Fachbegriffe definiert (2010, 38). Die beiden Wissenskategorien sind zwar verwandt, setzen sich aber nicht gegenseitig voraus. Die Untersuchung zur fachsprachlichen Organisation des mentalen Lexikons wird dadurch erschwert, dass die Grenzen zwischen gemeinsprachlichem und fachsprachlichem Wortschatz verschwommen sind (Fóris 2005, Kovács-Osvald 2010, 40). Dies gilt insbesondere für die Sprache des Sports, da die Fachbegriffe populärer Sportarten und anderer Sportarten auch für Nicht-Sportbegeisterte bekannt sind (Tor, Dribbling). 


\section{Konzeptualisierung, Bedeutungserklärung}

Die sprachliche Darstellung von Konzepten erfolgt in Begriffen. Terminologische Untersuchungen umfassen die Analyse von Konzepten (Wissensformen), die Beschreibung ihrer Haupt- und Nebenmerkmale sowie die Erstellung einer Definition und die Angabe der Bedeutung (Fóris 2014, 30). Der terminologische Ansatz der Fachsprachen ist konzeptuell, linguistisch und pragmatisch-kommunikativ geprägt.

Die Definition ist die Bestimmung der Bedeutung von Wörtern, die im Wesentlichen aus zwei Teilen besteht: dem zu definierenden Begriff einerseits und dem definierenden Teil andererseits. Bei der Bestimmung legt man die Hauptmerkmale des zu definierenden Begriffs oder Gegenstands fest und grenzt inn gegen alle anderen ab (Bóna-Imre 2017, 187). Die Definition funktioniert nur auf konzeptioneller Ebene, auf der Erfahrungsebene wird eine Klassifizierung vorgenommen.

Für die Definition ist die Kenntnis des Begriffs erforderlich, d.h. das Kind muss in der Lage sein, Fakten, Informationen, Begriffe, Regeln, Theorien abzurufen und zu erkennen, sowie die Zusammenhänge zu interpretieren und in eigenen Worten zu formulieren. Zu einem effektiven Lern- und Lehrprozess ist es notwendig, die Objekte auch auf einer interpretativen (Meta-) Ebene zu kennen, um erklären zu können, was was ist. Das heißt, wir müssen auch die Definition kennen, um eine klare Einstufung/Abgrenzung vornehmen zu können (Nagy 2003).

Auf der ersten Definitionsstufe kann das Kind verallgemeinerte Merkmale auflisten, auf der zweiten Ebene kann es allgemeine Begriffe auf bestimmte Situationen beziehen, und auf der dritten Ebene ordnet es sie in ein Begriffssystem ein (Nagy 1976, 254, Nagy-Varga 2014). Die Definition kann durch Angabe eines Synonyms erfolgen, sie kann aber auch auf Paraphrase, Beschreibung und Vergleich beruhen (Adamik és mtsai 2004, 313-316).

\section{Fachsprachengebrauch im Sportunterricht}

Die Sprache des Sports gehört zu jenen Fachsprachen, die in der letzten Zeit die größte Dynamik aufzeigen. Nachdem sich die gesellschaftliche Bedeutung des Sports im 20. Jahrhundert radikal verändert hatte (steigendes Gesundheitsbewusstsein, Zunahme der Rolle der aktiven und sinnvollen Freizeitgestaltung, Aufstieg vom Leistungssport zum Beruf und zum Lebensunterhalt, Erhebung vom Sport zu einem Mittel der Massenunterhaltung), entwickelte sich auch seine Sprache zu einer weit bekannten und verwendeten Fachsprache (Fóris-Bérces 2006). Sie steht in ständiger Interaktion mit der Gemeinsprache und unterscheidet sich von ihr vor allem im Wortschatz. Früher war die ungarische Sportsprache das wirksamste Mittel zur Massenverbreitung von Sport und zum Empfang von sportbezogenen Botschaften der Massenkommunikation (Fóris-Bérces 2005).

Die Terminologie der offiziell registrierten traditionellen Sportarten ist inzwischen gut etabliert und findet sich in den Regelwerken der einzelnen Sportarten und in einigen Fällen in deren zwei- oder mehrsprachigen Wörterbüchern. Dank 
der Forschung wird auch das konzeptuelle und terminologische System neuerer Sportarten immer bekannter (Fóris-Bérces 2005, 2006, Mátis 2009, GrétsyBalázs szerk. 2003).

Die Hauptaufgabe des Bewegungsunterrichts ist die Aneignung von Handlungen, aber der Erfolg und die Effizienz des Lernens hängt auch in hohem Maße von den kognitiv-verbalen Komponenten ab, da es durch diese Komponenten ermöglicht wird, Wissen und Erfahrung im Zusammenhang mit den Handlungen zu speichern und abzurufen. „Angemessene und praktische Informationen über die Bewegungsausführung, die Nutzungsformen, die Nutzbarkeit des Gelernten, die Regeln usw. sind unentbehrlich für die lernfördernde Bewegungsvorstellung und das Wecken der Motivation“ (Bíróné 2011). Bewegungsformen können nur durch das mit Hilfe der Sprache erzeugte innere Bild, das Gedankenmodell erfasst und aufgezeichnet werden.

Das verbale Lernen und somit die Kenntnis der Terminologie der einzelnen Sportarten ist Teil der Fachsprache des Sports. Die differenziertere und präzisere Verwendung einer dem Alter und den Vorkenntnissen der Sportschüler angepassten Terminologie macht die Entwicklung der Bewegungsvorstellung und der Feinkoordination effizienter und fördert letztlich das Bewegungslernen (Bíróné 2011).

Die erste Phase des Bewegungsunterrichts ist die des Wissenstransfers und der Wissensverarbeitung, in der die Sportler neue Bewegungsformen und die für deren Aneignung notwendigen Begriffe kennenlernen und einen Einblick in die Fachsprache der jeweiligen Sportart erhalten (Szatmári 2009,686). Das Ziel in dieser Phase ist es, die richtigen Bewegungsmuster zu erlernen, wobei auch die verbale Kommunikation (Darstellung, Erklärung) eine wichtige Rolle spielt, aber die Wissensvermittlung kann durch ein visuelles Signalsystem (Präsentation, Einsatz audiovisueller Mittel) unterstützt werden. Die Kommunikation des Trainers ist durch Redundanz gekennzeichnet, um das Verständnis zu erleichtern. Der Reichtum seines verbalen und nonverbalen Repertoires wirkt sich auf die Effektivität der Kommunikation aus (Budainé 2016, 41).

Übungen helfen dabei, die richtige Bewegungsvorstellung zu etablieren, und ihre Rolle ist wichtig, um das Gelernte zu festigen und zu vertiefen. In diesem Fall wird die verbale Kommunikation reduziert (gezielte, kurze Anweisungen), und die nonverbale Kommunikation (wiederholte Darstellung) sowie die taktile (auf Berührung basierende) Kommunikation (Hilfe, Fehlerkorrektur) treten in den Vordergrund. In dieser Phase kommt der Fachsprache eine größere Rolle zu, da sie eine gezieltere und damit effektivere Kommunikation ermöglicht.

Beim Erlernen der Bewegungsformen und später in der zweiten Phase des motorischen Lernens ist es für die Sportler wichtig, Rückmeldung über die Bewegungsausführung zu erhalten, damit sie ihre Bewegungsmuster dementsprechend verändern oder verbessern können.

Im Schwimm- und Wasserballsport ist das Kommunikationsmedium außergewöhnlich, weil die Informationen, die durch Hören und Sehen wahrgenommen werden, durch das Wasser eingeschränkt werden: Der hohe Geräuschpegel im Schwimmbad dämpft das Gehör und das Wasser verringert die Sicht und damit 
die Konzentration (Bíró 2006). Daher spielt die Kongruenz, die sich gegenseitig verstärkende Harmonie zwischen verbaler und nonverbaler Kommunikation eine besondere Rolle. Im Schwimm- und Wasserballunterricht ist es wichtig, dass die Kinder die Begriffe kennen (z.B. Fußspitze, Eckwurf, Auszeit, Fußspitzen hochziehen), die für die Bewegungs- und Strategievermittlung wichtig sind, aber die Form des Lehrens/Lernens ist anders als im Klassenzimmer. Dem Gleichgewicht zwischen Erklärung und Vorführung kommt eine viel größere Rolle zu (Bíró 2006).

\section{Forschungshintergrund, Forschungsfragen, Hypothesen}

Ich habe meine Recherchen bei den Nachwuchsteams des Wasserballklubs in der ungarischen Stadt Eger durchgeführt. Dabei habe ich den Fachwortschatz der Kinder in der Altersgruppe von 9 bis 10 Jahren erfasst. Meine Informanten waren Jungen. Die Kinder dieser Altersgruppe sind seit knapp einem Jahr Mitglieder der Juniorenmannschaft (nach mindestens zwei Jahren Grundausbildung). Die Gesamtzahl der Teilnehmer in der Gruppe betrug 41, an der Umfrage nahmen 26 Personen teil. Eine Schwierigkeit bestand darin, dass einige Mitglieder jeden Tag zum Training gehen (5 Nachmittage pro Woche), andere jedoch weniger häufig (normalerweise 3 Nachmittage). Die Zahl der Abwesenheiten kann auch aus anderen unvorhergesehenen Gründen (Krankheit, schulische oder familiäre Aktivitäten) höher sein.

Meine Untersuchung basierte auf Begriffen in den Wasserballregeln. Ich bat die Kinder, 10 Begriffe (Substantive) zu definieren (am Spiel beteiligte Personen, Wurfarten, Spielerfehler, Strafen). Bei den Definitionen habe ich berücksichtigt, wie gut das Kind den jeweiligen Begriff kennt und welche Definitionsstrategie angewendet wird. Manchmal, wenn ich eine komplexe Antwort erhielt, konnte ich die Definition in mehrere Typen einteilen. Die nach Nagy-Varga verwendeten Kategorien waren die von Judit Bóna und Angéla Imre benannten Definitionsstrategien:

a) Überbegriff: Bezeichnung einer Kategorie, die größer ist als das zu definierende Wort;

b) Teil-Ganzes-Beziehung: Ausdruck dessen, 1. was Teil eines anderen ist; 2. woraus ein Gegenstand besteht, welche Teile er hat;

c) Beispiel: Nennung eines konkreten Einzelfalls/Typs/Musters statt einer Definition;

d) Synonym: Angabe eines anderen Wortes mit gleicher Bedeutung;

e) Gegenteil: Verwendung eines Wortes mit gegenteiliger Bedeutung;

f) Funktion: Angabe dessen, wofür bestimmte Gegenstände verwendet werden;

g) Umschreibung: Ausdrucksformen vom Typ derjenige, der... ohne Nennung einer Hauptkategorie;

h) persönliche Einstellung: Erwähnung positiver oder negativer Erfahrungen, Gefühle;

i) Handlungen (bei Verben): Darstellung einer Bewegung;

j) Angabe einer grammatikalischen Kategorie: Erwähnung der Wortart des jeweiligen Begriffs; 
k) keine Antwort.

Da ich nicht nur an der Kenntnis der Terminologie interessiert war, sondern auch daran, wie ihr Erwerb erfolgt und inwiefern die Kenntnis der Begriffe die Wirksamkeit des Unterrichts beeinflusst, nahm ich als Beobachterin an offenen Trainings teil, um das Ergebnis zu kontrollieren und eine differenziertere Schlussfolgerung zu formulieren, und führte strukturierte Interviews mit den Trainern durch. Die Hauptmotivation für die Anwendung einer gemischten Methodik bestand darin, die Forschungsfrage so vollständig wie möglich beantworten zu können und fundiertere Schlussfolgerungen zu ziehen.

Ich gehe davon aus, dass die Kinder (1) bereits Begriffe kennen, die nicht nur im Wasserball vorkommen, und (2) dass für die Definition am häufigsten auf Beispiele zurückgegriffen wird. Eine weitere Hypothese war, dass die Fachbegriffe aufgrund des speziellen Kommunikationsmediums (3) häufiger verwendet werden, weil sie die Kommunikation kompakter machen können.

\section{Ergebnisse}

In 50 Fällen erhielt ich von den Kindern die Antwort „Ich weiß es nicht“, oder sie gaben keine auswertbare Erklärung ab. Die meisten von ihnen kannten den Begriff „Mannschaftskapitän“ (23 gaben die richtige Antwort), und nur die wenigsten (10 Kinder) konnten „Spielzeit“ nicht definieren. Ein Grund dafür mag sein, dass „Mannschaftskapitän“ nicht nur ein im Wasserball verwendter Begriff ist, und „Spielzeit“ erst bekannt wird, wenn die Kinder die Wettkämpfe als Zuschauer verfolgen oder als Sportler bestreiten.

Die am häufigsten verwendete Definitionsstrategie - mit einem hohen Anteil an jedem Begriff - war die Umschreibung (49\% der eingegangenen Antworten). Als Umschreibung gelten Definitionen, die im Ungarischen mit Az, amikor..., Olyan, amikor..., Az, aki..., Az, ha... beginnen, ohne dabei eine größere Kategorie zu benennen (Nagy-Varga 2014). Zum Beispiel: (Sarokdobás „az, amikor a kapusról kimegy a labda, az ellenfél dobhatja; az után löjük, hogy a kapus kezéről a kapu mögé esik a labda”) Eckwurf „ist, wenn der Ball vom Torhüter ins Aus geht; den Eckwurf bekommt der Gegner; auf Eckwurf wird entschieden, wenn der Ball von der Hand des Torhüters hinter das Tor fällt". Oder bei der Definition von „Mannschaftskapitän“: (az, aki megmondja meccs közben, hogy mit csinálj) Person, die uns sagt, was wir während des Spiels machen sollen. Die Definition dieses Begriffs zeigt das unterschiedlichste Bild: 2 von 26 Befragten kannten ihn nicht, 1 gab keine auswertbare Antwort, und die anderen 23 Definitionen konnten entlang von drei Strategien eingeteilt werden:

- Umschreibung (Person, die die Mannschaft anführt und sagt, was man während des Spiels machen soll),

- Übergeordneter Begriff, d.h. Benennung einer größeren Kategorie mit individuellen Unterscheidungsmerkmalen (Person, die das Team anführt; Teammitglied an der Spitze eines Teams) oder komplexe Antwort (Person, die der Kapitän des Teams ist, und deren Aufgabe es ist, das Team zusammenzuhalten) 
- Beispiel in einer komplexen Antwort (wer das Team im Wasser anführt, er gibt zum Beispiel die Aufstellungen vor).

Die Umschreibung als Definitionsstrategie war häufig bei der Bestimmung von „Zeitspiel“ (genutzt von 18 Personen): wenn der Spieler den Ball nicht wegwirft; wenn ein Spieler den Ball lange in der Hand hält; wenn jemand den Ball nicht abgibt.

Relativ hoch war auch die Zahl der Begriffe, die durch Synonyme definiert wurden (22\%). Am häufigsten wurden sie bei der Definition von „Strafwurf“" (Viermeter, Fünfmeter) oder „Eckwurf“ (Ecke) sowie bei Verhinderungen (Block, Abwehr) angewendet.

In einigen Fällen wurde die Antwort durch ein Beispiel veranschaulicht, am häufigsten bei „Freiwurf“: (amikor például lenyomnak, akkor dobhatjuk; amikor lefaultolnak, akkor a másik csapat dobhatja) Es gibt einen Strafwurf, wenn ich unter Wasser gedrückt werde; Beim Foul bekommt ihn die andere Mannschaft. Eine solche Strategie gibt es auch bei der Definition von „Strafwurf": (például ha a kettesen belül lenyomnak; például, amikor a büntetőterületen belül lenyomnak, illetve az időhúzásnál: passzolgatunk például a meccs végén, amikor nyerésre állunk) Wenn man mich zum Beispiel innerhalb des Zwei-Meter-Raumes unter Wasser drückt; wenn man mich etwa innerhalb des Strafraums unter Wasser drückt, und bei „Zeitspiel“: Am Ende des Spiels lassen wir einander den Ball zuspielen, wenn die Zeichen auf Sieg stehen.

Die anderen oben erwähnten Definitionstypen kommen nur selten vor. Die Definition durch die Funktion wird meist bei der Bestimmung von Gebrauchsgegenständen angewendet, d.h. wenn die Kinder erklärten, wie ein Gegenstand verwendet wird. In dem vorliegenden Kontext wird Funktion verwendet, wenn die Rolle der am Spiel beteiligten Personen definiert wird: (a gólbíró feladata, hogy vitatható helyzetekben döntsön; amikor nem tudnak döntetni, hogy gól-e vagy nem, az ö feladata, hogy megmondja) Die Aufgabe des Torrichters ist es, in strittigen Situationen zu entscheiden; wenn nicht entschieden werden kann, ob der Ball im Tor war, soll er entscheiden. Auch die Teil-Ganzes-Beziehung kommt nur ab und zu zum Einsatz. Sie kann entweder ausdrücken, dass etwas Teil eines anderen ist, oder aus welchen Teilen etwas besteht und was sein Inhalt ist. So zum Beispiel bei der Bestimmung von „Spielzeit“: Sie besteht aus 4x8 Minuten.

\begin{tabular}{|l|l|}
\hline Strategie & Verwendungsrate \\
\hline Umschreibung & $49 \%$ \\
\hline Synonym & $22 \%$ \\
\hline Beispiel & $25 \%$ \\
\hline Funktion & $1 \%$ \\
\hline Komplexe Antwort & $7 \%$ \\
\hline Keine Antwort und falsche Antwort & $30 \%$ \\
\hline Teil-Ganzes-Beziehung & - \\
\hline Tabelle 1: Verwendete Definitionsstrategien
\end{tabular}


Der Erwerb der Terminologie durch die Kinder erfolgt schrittweise entsprechend ihrer kognitiven Reife. Der Unterrichtsprozess lässt sich in zwei Phasen unterteilen: die Phase des Wissenstransfers und der Verwendungsphase Bíró 2007, 26). In der ersten Phase werden typische Begriffe einer Sportart gelernt, entweder durch neue Bewegungen, die von den Kindern nachgeahmt werden, oder durch die Darstellung neuer Situationen.

Dem angemessenen Sprachgebrauch, der Verwendung sportspezifischer Terminologie kommt auch später, bei der Formulierung von Anweisungen und Rückmeldungen in der Übungsphase eine wichtige Rolle zu. Aufgrund des besonderen Mediums im Wassersportunterricht - das Wasser dämpft das Gehör, der Lärm im Schwimmbad erschwert das Verstehen - ist es wichtig, dass sich der Trainer möglichst präzise ausdrückt. Natürlich spielen die nonverbalen Symbole, die die verbale Kommunikation begleiten und verstärken, eine herausragende Rolle in der Kommunikation: Mimik, Gestik, Körperhaltung und Taktilität (Berührung). Berührung ist im Sport, insbesondere in der Sportpädagogik, von grundlegender Bedeutung. Während im traditionellen Klassenzimmer der Körperkontakt zwischen Lehrer und Schüler selten ist, da Berührungen in der Regel ein Maß an Intimität erfordern, das mit der formalen Situation nicht vereinbar ist, ist dies für die Vermittlung und Verbesserung von Bewegungsmustern sowie die Förderung unentbehrlich. Ein kombinierter Einsatz macht nicht nur den Erwerb, sondern auch die Konsolidierung von Wissen effizienter.

Bei der Beherrschung der Terminologie und der Vertiefung der Regelkenntnisse spielen die ersten Meisterschaftsspiele eine wichtige Rolle, bei denen die Kinder die sportspezifischen Fachwörter in konkreten Wettkampfsituationen kennenlernen können. Auch Experten betonen die Bedeutung des Erfahrungslernens bei diesen Wettkämpfen. Der Erfolg wird hier nicht an Toren gemessen, sondern an erlebten Situationen, den vermittelten Regeln und der Entwicklung der motorischen Fähigkeiten.

\section{Zusammenfassung}

Verbalität spielt beim Erlernen von Bewegungshandlungen eine ebenso wichtige Rolle wie in anderen Lernprozessen. Die Verwendung einer dem Alter, der kognitiven Reife und dem Sportwissen angemessenen Fachsprache, die präzise und angemessene Form der Kommunikation, kombiniert mit geeigneten Bewegungsmustern, führt zu leichter abrufbarem Wissen.

Das Ziel meiner Forschung war es, Veränderungen in den Fachsprachenkenntnissen, im Fachwortschatz von Sport treibenden Kindern zu erfassen. Ich habe meine Umfrage unter Wasserball spielenden Kindern im Nachwuchsalter ( 9 bis 10 Jahre) durchgeführt. Meine ursprünglichen Hypothesen wurden bestätigt, da die im Alltag verwendeten Begriffe, die auch für andere Sportarten typisch sind, bekannter waren. Die am häufigsten gewählte Definitionsstrategie war die Umschreibung. Meine Beobachtungen bestätigten im Einklang mit den Aussagen der 
Trainer, dass die Natur des Sports und das Kommunikationsmedium eine reduzierte, zielgerichtete verbale Kommunikation erfordern, die durch eine angemessene Verwendung von Terminologie unterstützt werden kann.

Meine Ergebnisse können zusätzliche Erkenntnisse für die Forschungen zur Kindersprache und Sportpädagogik liefern.

\section{Literatur}

Ablonczyné Mihályka Lívia (2006): Gazdaság és nyelv. Pécs: Lexikográfia Kiadó.

Adamik Tamás-A. Jászó Anna-Aczél Petra (2004): Retorika. Budapest: Osiris Kiadó.

Adamikné Jászó Anna (2006): A nyelvi tudatosság fejlödése az anyanyelv elsajátitása során. Alkalmazott Nyelvtudomány 6/ 1-2, 5-23. p.

Bíró Melinda (2006): Tanítási-tanulási stratégiák a mozgásos cselekvéstanitás speciális területén, az úszásoktatásban. Új Pedagógiai Szemle 2006/9 Letölthetö: https://folyoiratok. ofi.hu/uj-pedagogiai-szemle/tanitasi-tanulasi-strategiak-a-mozgasos-cselekvestanitas-specialis-teruleten-az (Utolsó letöltés: 2020. december 8.)

Bíróné Nagy Edit (szerk.) (2011): Sportpedagógia. Budapest-Pécs: Dialóg Campus Kiadó. Letölthetö: http://eta.bibl.u-szeged.hu/1618/1/sportpedagogia.pdf (Utolsó letöltés: 2021. január 14.)

Bóna Judit-Imre Angéla (2017): Szójelentések meghatározása 5 és 9 éves kor között. Beszédkutatás 25, 185-203. p.

Csépe Valéria-Györi Miklós-Ragó Anett szerk. (2007): Általános pszichológia 2. Tanulás, emlékezés, tudás. Budapest: Osiris Kiadó.

Fóris Ágota (2014): A szaknyelvek terminológiai szempontú megközelítése. In: Veszelszki Ágnes-Lengyel Klára (szerk.) Tudomány, technolektus, terminológia - a tudományok, szakmák nyelve. Budapest: Éghajlat Könyvkiadó. 27-36. p.

Fóris Ágota-Kováts Anna-Póla Péter (2003): Nyelvi és szaknyelvi oktatás egy felmérés tükrében. In: Tóth Szergej (szerk.) Nyelvek és kultúrák találkozása. Szeged: Szegedi Tudományegyetem JGYTFK. 157-162. p.

Fóris Ágota-Bérczes Edit (2005): Sport, gazdaság, terminológia. Tudásmenedzsment VI. évf. 2: 117-127. p.

http://epa.oszk.hu/02700/02750/00012/pdf/EPA02750_tudasmenedzsment_2005_02_117-127.pdf (Utolsó letöltés: 2020. december 15.)

Gósy Mária 2005. Pszicholingvisztika. Budapest: Osiris Kiadó.

Kovács László-Osvald Mária (2010): A mentális lexikon szakmai szerveződése. Modern nyelvoktatás. 2010/2-3, 38-46. p.

Kurtán Zsuzsa 2011. Pedagógiai nyelvhasználat tudományos szövegekben. Iskolakultúra 2011/10-11: 8-17. p.

Mátis Bernadett (2009): Sportnyelvünk változásai a 21. század elején - labdajátékok szókészletének összehasonlító elemzése. PhD disszertáció. http://doktori.btk.elte.hu/ lingv/matisbernadett/diss.pdf (Utolsó letöltés: 2021. január. 12.)

Nagy József (2003): A rendszerező képesség fejlődésének kritériumorientált feltárása. Magyar Pedagógia 103, 269-314. p. 
Nagy-Varga Zsolt (2014): Definícióalkotási stratégiák tizenéves diákok körében. Anyanyelvpedagógia 2014/3. http://www.anyp.hu/cikkek.php?id=525 (Utolsó letöltés: 2020. október 1.)

Neuberger Tilda (2008): A szókincs fejlődése óvodáskorban. Anyanyelv-pedagógia, 2008/3-4. http://anyanyelvpedagogia.hu/cikkek. php?id=86 (Utolsó letöltés: 2021. január 20.)

Neuberger Tilda (2017): A szókincs alakulása a beszédfejlődésben. In: Bóna, Judit (szerk.) Új utak a gyermeknyelvi kutatásokban. Budapest: ELTE Eötvös Kiadó. 121-139. p.

Szatmári Zoltán (2009): Az oktatás értelmezése a testnevelésben. In: Szatmári, Zoltán (szerk.) Sport, életmód, egészség. Budapest: Akadémiai Kiadó. 675-680. p.

Budainé Csepela Yvette (2016): Az edző-sportoló kapcsolat - mint sajátos pedagógiai helyzet - jellegzetességei. In: Hamar Pál szerk. A mozgás mint személyiségfejlesztő tényező. Budapest: Eötvös József Könyvkiadó.

Nemzeti alaptanterv (NAT) 2012. Magyar Közlöny 66, 10673- 10847. p. Letölthetö: https://ofi.oh.gov.hu/sites/default/files/attachments/mk_nat_20121.pdf 